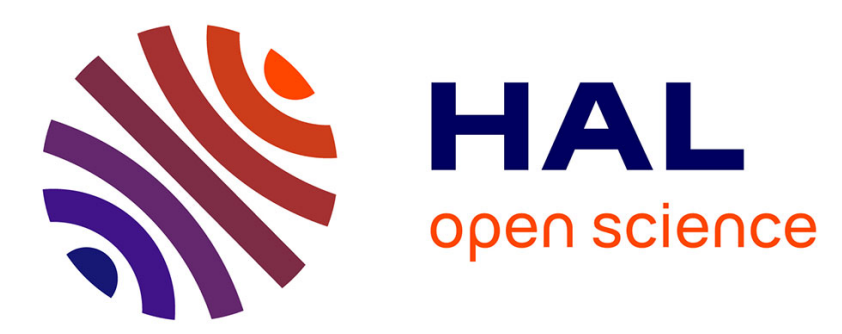

\title{
Low red cell production may protect against severe anemia during a malaria infection - insights from modeling
}

\author{
Deborah Cromer, Jaroslav Stark, Miles P Davenport
}

\section{To cite this version:}

Deborah Cromer, Jaroslav Stark, Miles P Davenport. Low red cell production may protect against severe anemia during a malaria infection - insights from modeling. Journal of Theoretical Biology, 2009, 257 (4), pp.533. 10.1016/j.jtbi.2008.12.019 . hal-00554553

\section{HAL Id: hal-00554553 \\ https://hal.science/hal-00554553}

Submitted on 11 Jan 2011

HAL is a multi-disciplinary open access archive for the deposit and dissemination of scientific research documents, whether they are published or not. The documents may come from teaching and research institutions in France or abroad, or from public or private research centers.
L'archive ouverte pluridisciplinaire HAL, est destinée au dépôt et à la diffusion de documents scientifiques de niveau recherche, publiés ou non, émanant des établissements d'enseignement et de recherche français ou étrangers, des laboratoires publics ou privés. 


\section{Author's Accepted Manuscript}

Low red cell production may protect against severe anemia during a malaria infection - insights from modeling

Deborah Cromer, Jaroslav Stark, Miles P Davenport

PII: $\quad$ S0022-5193(08)00655-3

DOI: doi:10.1016/j.jtbi.2008.12.019

Reference: YJTBI 5404

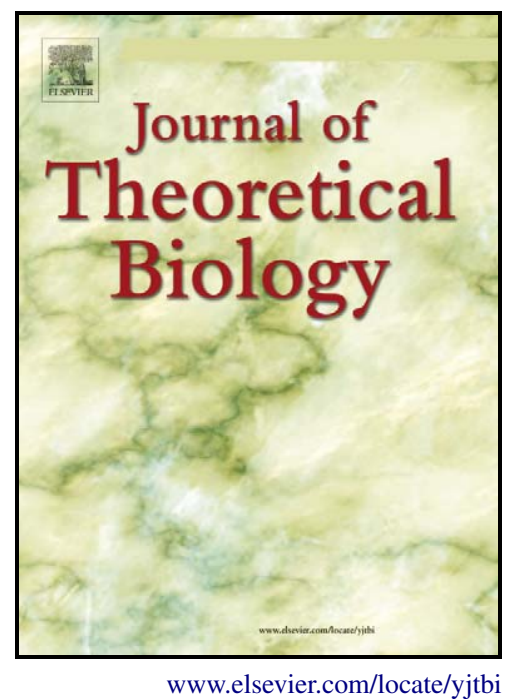

To appear in: $\quad$ Journal of Theoretical Biology

Received date: 23 April 2008

Revised date: $\quad 9$ December 2008

Accepted date: 9 December 2008

Cite this article as: Deborah Cromer, Jaroslav Stark and Miles P Davenport, Low red cell production may protect against severe anemia during a malaria infection - insights from modeling, Journal of Theoretical Biology (2008), doi:10.1016/j.jtbi.2008.12.019

This is a PDF file of an unedited manuscript that has been accepted for publication. As a service to our customers we are providing this early version of the manuscript. The manuscript will undergo copyediting, typesetting, and review of the resulting galley proof before it is published in its final citable form. Please note that during the production process errors may be discovered which could affect the content, and all legal disclaimers that apply to the journal pertain. 


\section{LOW RED CELL PRODUCTION MAY PROTECT AGAINST SEVERE ANEMIA DURING A MALARIA INFECTION - INSIGHTS FROM MODELING.}

Deborah Cromer ${ }^{1,2,3^{*}}$, Jaroslav Stark ${ }^{1,2}$ and Miles P Davenport ${ }^{3}$

${ }^{1}$ Department of Mathematics, Imperial College London, London SW7 2AZ, UK.

${ }^{2}$ Centre for Integrative Systems Biology at Imperial College (CISBIC), Imperial College London, London SW7 2AZ, UK.

${ }^{3}$ Department of Haematology, Prince of Wales Hospital and Centre for Vascular Research, University of New South Wales, Kensington NSW 2052, Australia.

" To whom correspondence should be addressed:

Department of Mathematics

Imperial College, London

SW7 2AZ

UK

ph: +44 (0)20 75948483

fax: +44 (0)20 75948517

d.cromer05@imperial.ac.uk

\section{SuPPlemEnTal Data}

This article contains supplemental data. 


\begin{abstract}
The malaria parasite causes lysis of red blood cells, resulting in anemia, a major cause of mortality and morbidity. Intuitively, one would expect the production of red blood cells to increase in order to compensate for this loss. However, it has been observed that this response is weaker than would be expected. Furthermore iron supplementation for iron deficient children in malaria endemic regions can paradoxically adversely affect the clinical outcome of malaria infection. A possible explanation may lie in the preference that some malaria parasites show for infecting immature red blood cells (reticulocytes). In the presence of a parasite preference for immature red cells, a rise in red cell production can 'fuel the fire' of infection by increasing the availability of the parasite's preferred target cell.

We present a mathematical model of red blood cell production and infection in order to explore this hypothesis. We assess the effect of varying the reticulocyte replacement rate and preference of the parasite for reticulocytes on four key outcome measures assessing anemia and parasitemia.

For a given level of parasite preference for reticulocytes we uncover an optimal erythropoietic response which minimizes disease severity. Increasing red blood cell production much above this optimum confers no benefit to the patient, and in fact can increase the degree of anemia and parasitemia. These conclusions are consistent with epidemiological studies demonstrating that both iron deficiency and anemia are protective against severe malaria, whilst iron supplementation in malaria endemic regions is with an increased number of malaria related adverse effects. Thus, suppression of red blood cell production, rather than being an unfortunate side effect of inflammation, may be a host protective effect against severe malarial anemia.
\end{abstract}

\title{
KEYWORDS
}

Reticulocytes, Erythropoiesis, Mathematical, Hematopoiesis, Iron, Red Cells 


\section{INTRODUCTION}

Malaria presents a major health burden in the developing world, and is associated with a range of clinical symptoms including acute and chronic anemia. The malaria parasite causes lysis of both infected and uninfected red blood cells (RBCs) (Dondorp et al., 1999; Ekvall, 2003), leading to anemia, one of the most significant clinical outcomes of the disease. Intuitively, one would expect the organism to respond by increasing the production of RBCs to compensate. Instead it has been observed in that in both human (Abdalla et al., 1980; Abdalla, 1990) and animal (Chang et al., 2004b; Egan et al., 2002; Maggio-Price et al., 1985) malarias the RBC replacement rate may be insufficient to compensate for the degree of anemia. It is usually thought that this suppression of the erythropoietic response to anemia may be an unfortunate side effect of the inflammatory response to infection. One would expect this to exacerbate and prolong the anemia and hence potentially have an adverse effect on the prognosis. It has been postulated that an inadequate erythropoietic response contributes to disease severity and death.

This apparent paradox is reinforced by observations that in regions where malaria is endemic, iron supplementation for iron-deficient children may have significant detrimental consequences on mortality and morbidity during malaria infection (English and Snow, 2006; Sazawal et al., 2006; Smith et al., 1989). This is despite the fact that iron supplementation improves hematological and iron status. In regions where malaria is not endemic, iron supplementation has no adverse effects (Iannotti et al., 2006; Oppenheimer, 2001). A separate study (O'Donnell et al., 2007) has investigated the relationship between anemia and erythropoietin (EPO) production and demonstrated that younger children have higher EPO production for a given level of anemia. Since severe malarial anemia (SMA) is more common at early ages when there is a high level of erythropoietin (EPO) produced in response to anemia, this suggests that high RBC production may have a paradoxical negative effect on anemia during malaria.

A possible explanation comes from the preference exhibited by many species of malaria for immature RBCs (reticulocytes) compared to mature RBCs (erythrocytes). This preference is estimated to be as high as 34-180 fold in P. vivax (Mons, 1990; Mons et al., 1988a; Mons et al., 1988b), and in the range 1.6-14 fold for P. falciparum (Hegner, 1938; Pasvol et al., 1980; Wilson et al., 1977). In the presence of such preference, a high reticulocyte replacement rate (RRR) could serve to provide large numbers of the preferred target cells for the parasite. This could significantly boost parasitemia and increase the severity of the disease. Iron supplementation in iron-deficient individuals would be likely to increase reticulocyte production leading to similar effects.

On the other hand, a high RRR will also serve to replace those RBCs destroyed by the parasite, serving to alleviate anemia and hence having a positive effect on the outcome of the in- 
fection. The picture is further complicated by the results of experiments that manipulated reticulocyte production in mice (Singer, 1954a) and rats (Zuckerman, 1957) infected with $P$. berghei, and more recently in mice infected with P. chabaudi (Chang et al., 2004a). An increase in reticulocyte production early in infection can significantly increase parasitemia and reduce survival rates. On the other hand, an increase in reticulocyte production at an appropriate stage of the infection led to reduced mortality (Chang et al., 2004a).

The overall effect of an increase in reticulocyte replacement rate thus depends on the balance and timing of these positive and negative interactions and on the degree of the parasite preference for reticulocytes, which varies from species to species. It is impossible to infer the integrated outcome of all these factors by intuition alone. It may thus be that erythropoietic suppression is simply an inadvertent consequence of inflammation and has negative consequences for the host, or it may be a host protective effect to reduce the availability of preferred target cells.

Similar counter-intuitive phenomena that cannot be understood through conventional experimental approaches are routinely encountered in biological systems. One common reason why intuition may be insufficient to understand such behaviour is the presence of regulatory mechanisms acting in opposing directions. The outcome of these mechanisms will depend on the fine balance between competing pressures (in this example the response to anemia competes with the production of preferred targets), and this balance may be a complex function of a wide range of other interactions. The only way to predict and explain the behaviour of such systems is to quantify the key interactions and analyse them within the framework of a mathematical or computational model (Hardy and Stark, 2002; Hastings and Palmer, 2003; Stark and Hardy, 2003).

The majority of models of pathogen growth within a host are straightforward ODE models, where target cells are treated as homogeneous. To model a preference for target cells of a certain type, one must incorporate into the model the variable for which the pathogen shows its preference. Thus, in this instance we must structure our model by red blood cell age. The model developed here does this through the use of a continuous age variable, and has a similar structure to the model presented by $\{$ Antia, 2008 \#302\} which discuss the role of RBC resourse limitation in rodent malaria. Such a continuous age variable has the effect of turning the model into a complex integro-differential equation. This is in contrast to an earlier age structured model by McQueen and McKenzie which uses discrete age compartments and was applied to analyse the difference between the dynamics of various strains of malaria (McQueen and McKenzie, 2004). The detailed differences between the two models are discussed in the materials and methods section. 
Our mathematical model allows us to distinguish between competing pressures and to explore the effects of changing key parameters such as the parasite preference for reticulocytes and the rate of reticulocyte production. This allows us to understand the circumstances under which decreased reticulocyte production is either beneficial or harmful to the host. Extensive numerical investigation suggests that in the absence of any preference of the parasite for reticulocytes an elevated RRR is always beneficial to the host, since it allows rapid replacement of destroyed RBCs. However, we confirm that in the presence of a preference of the parasite for younger cells, a high RRR is not always advantageous. In fact, for a given reticulocyte preference there is a threshold RRR beyond which increasing the RRR becomes detrimental. This threshold RRR is usually far below the normal RRR in response to anemia, suggesting an important benefit of erythropoietic suppression.

\section{Materials AND Methods}

The model used here is based on one previously described in (Cromer et al., 2006), but has been expanded to incorporate the dynamics of merozoite release and invasion. The parameters of the model have also been altered to values that are appropriate for humans rather than mice. The model captures the continuous birth and death processes of cells as well as the discrete process of parasite rupture, but differs from the earlier model in the way that parasitized cells are produced. We introduce a continuous process to describe parasite growth, and hence provide a self contained model of the cell populations during a malarial episode. This can be used to make predictions about the course of an infection. In the previous model we considered the physiological relationship between reticulocyte replacement rate and red blood cell levels. In this model we allow the reticulocyte replacement rate to vary from its baseline production up to a normal erythropoietic response. This allows us to investigate the effects of reticulocyte production in response to anemia.

The model describes the population dynamics of classes of parasitized and unparasitized cells, separated into reticulocytes and erythrocytes (Figure 1). Cells move between the groups according to the following interactions:

i) Reticulocytes are produced according to a production function that is dependant on the RBC levels 6 days earlier (Belair et al., 1995).

ii) Reticulocytes mature into erythrocytes after one reticulocyte lifetime, $l_{r}=2.8$ days (Colijn and Mackey, 2005).

iii) Unparasitized reticulocytes and erythrocytes can become parasitized and move into their respective parasitized classes. The rate of infection is determined by the number of available merozoites, and the preference of merozoites for erythrocytes or reticulocytes. 
The infection rate of the parasite in mature erythrocytes is $g$ and infection rate in reticulocytes is $g p$ where $p$ is the reticulocyte preference.

iv) All cells are cleared according to an exponential decay function with the natural decay rate required for baseline turnover of $\mathrm{RBCs}$ that equates to an average $\mathrm{RBC}$ lifetime of 120 days (Belair et al., 1995).

v) Parasitized cells rupture one parasite lifetime $l_{p}=2$ days (Garnham, 1966) after becoming infected, releasing a number of free merozoites (number of released merozoites is 16), which have a short lifespan (20 minutes) unless they infect another cell (Anderson et al., 1989).

Simplified equations describing the model are given in equations 1-6 below. An explanation of the terms in the equations are given in A more in depth derivation including details of model implementation is given in the Supplementary Online Materials (SOM). Variables in the model are listed in Table 1. Parameters values are taken from previously published experimental data, (Supplementary Table 2).

Our model has some similarities to that of McQueen and McKenzie (McQueen and McKenzie, 2004). Their model incorporated age restricted parasite invasion (parasites exclusively invade cells of a certain age) and an elevated red cell response to anemia. However, there are also a number of differences between the two models. We allow for parasites to have a (variable) preference for reticulocytes rather than being simply restricted in their invasion to cells of a specific age as was the case in the McQueen and McKenzie model. Such a preference is similar to what has been reported in in-vivo and in-vitro studies (Mons, 1990; Pasvol et al., 1980) and enables us to consider disease severity as a function of parasite preference for reticulocytes. A second difference lies in the reticulocyte replacement functions used. McQueen and McKenzie used only two replacement rates (basal production and an increase up to twice the basal rate), and did not attempt to model a 'physiological' response to different levels of anemia. Previous work has shown that an increase up to 10 times the basal rate is possible during severe anemia (Mackey, 1997a). In this paper we consider a range of different erythropoietic responses up to this maximum level. The significant flexibility that our model allows both in terms of parasite preference and reticulocyte production means that we are able to consider disease progression as a function of these two variables. The three dimensional plots shown in Figure 6 and Supplementary Figures 1-3 indicate the power of our model, and would have been difficult to generate using the model of McQueen and McKenzie.

In addition to the differences described above, there is also a conceptual difference between our model, and that of McQueen and McKenzie. In their model they allow cells to pass through different age compartments at a certain rate. At each point in time there is a probabil- 
ity that a cell will age from one compartment into the next Although this can give the correct average lifetime of cells, a finite number of cells will move through the compartments infinitely fast, and so age instantaneously. Using their model it would therefore be possible (and probable) that some newly created cells would immediately pass through the reticulocyte stage, and so not be subject to preferential infection.

Within the framework of our model cells age in a deterministic fashion. All cells must spend a specified amount of time as a reticulocyte (and so be the subject to preferential infection) before they age into an erythrocyte. Since a main priority of this paper is to accurately capture the preference of the parasite for newly created cells we felt that the potential instantaneous aging of cells in the McQueen and McKenzie model could impact on our results. We therefore chose to implement a model in which cells age in a deterministic fashion.

\section{Altering the Preference for Reticulocytes}

By changing the value of the reticulocyte preference $p$ we alter the probability that a free merozoite will invade a reticulocyte rather than an erythrocyte. A preference of 5 indicates that a merozoite is 5 times as likely to invade a reticulocyte as an erythrocyte.

\section{Modifying the Reticulocyte Replacement Rate}

The production of reticulocytes in response to reduced $\mathrm{RBC}$ levels is described by the function $f_{R}(t)$. The normal RRR is similar to that used in our earlier model (Cromer et al., 2006) and is a sigmoid curve that is dependant on the RBC level six days earlier (Belair et al., 1995). This RBC level is represented by the sum of all RBC populations $H(t)$. We have modified $f_{R}(t)$ slightly from (Cromer et al., 2006) to agree with literature estimates in other species (Mackey, 1997b) so that

$$
f_{R}(t)=f_{R}(H(t-6))=H_{0} \frac{\theta^{n}}{H(t-6)^{n}+\theta^{n}}
$$

We then modified this function to represent reduced reticulocyte replacement rates, yielding the sigmoid curves shown in Figure 2. In doing this, we maintained the same baseline RRR of $4.17 \times 10^{7}$ cells ml ${ }^{-1}$ day $^{-1}$ when the RBC count is at normal level $\left(5 \times 10^{9}\right.$ cells ml $\left.{ }^{-1}\right)$. This corresponds to the rate at which reticulocytes are produced to replace cells which are lost due to the natural aging of cells. The normal RRR has a maximum production rate of 10 times the baseline rate, attained when RBC levels drop to low values (Mackey, 1997b). Each modified $\mathrm{RRR}$ is defined by reducing the maximum production rate at low RBC numbers (Figure 2), expressed as a fold change relative to the baseline rate (detailed definitions are given in SOM). 


\section{Basic Equations of the Model}

The model can be understood through six basic equations (Equations 1-6). These include one equation (above) to describe the production of newly formed reticulocytes and five differential equations (below) which describe the change in populations of the five species in the model. Each term in the equations can be associated with one of the arrows of Figure 1.

$$
\begin{array}{lll}
\frac{d R_{u}}{d t} & = & f_{R}(t)-R_{u, \text { mat }}(t)-R_{u, \text { para }}(t)-R_{u, \text { decay }}(t) \\
\frac{d E_{u}}{d t} & = & R_{u, \text { mat }}(t)-E_{u, \text { para }}(t)-E_{u, \text { decay }}(t) \\
\frac{d R_{p}}{d t} & = & R_{u, \text { para }}(t)-R_{p, \text { mat }}(t)-R_{p, \text { rupt }}(t)-R_{p, \text { decay }}(t) \\
\frac{d E_{p}}{d t} & = & E_{u, \text { para }}(t)+R_{p, \text { mat }}(t)-E_{p, \text { rupt }}(t)-E_{p, \text { decay }}(t) \\
\frac{d M}{d t} & = & M_{\text {created }}(t)-M_{\text {used }}(t)-M_{\text {decay }}(t) \\
& = & m_{r}\left(R_{p, \text { rupt }}(t)+E_{p, \text { rupt }}(t)\right)-\left(R_{u, \text { para }}(t)+E_{u, \text { para }}(t)\right)-\delta_{m} M(t)
\end{array}
$$

These equations are defined in terms of two other quantities, $s\left(t_{b}, t\right)$ and $c\left(t_{b}, t_{p}, t\right)$. The first, $s\left(t_{b}, t\right)$, represents cells which were born at time $t_{b}$ and are still present and unparasitized at time $t$ and satisfies equation 7 , while $c\left(t_{b}, t_{p}, t\right)$ represents the number of parasitized cells born at time $t_{b}$, parasitized at time $t_{p}$ at still present at time $t$ and satisfies equation 8 . Note that where $\delta_{u}$ and $\delta_{p}$ are the decay rates of unparasitized and parasitized cells respectively.

$$
\begin{array}{ll}
\frac{\partial s\left(t_{b}, t\right)}{\partial t} & =-\delta_{u} s\left(t_{b}, t\right)-c\left(t_{b}, t, t\right) \\
\frac{\partial c\left(t_{b}, t_{p}, t\right)}{\partial t} & =-\delta_{p} c\left(t_{b}, t_{p}, t\right)
\end{array}
$$

\section{RESUlTS}

\section{Reticulocyte Preference Can Adversely Affect the Outcome of Infection}

We first examined the effects of changing the reticulocyte preference in the presence of a normal reticulocyte response (Figure 3A). In the absence of any reticulocyte preference, our model leads to target cell limited growth of the parasite (Phillips, 1996). Parasite numbers increase until the uninfected RBC count drops low enough to reduce the reproductive ratio of the parasite below one. Parasite levels then drop and RBCs are replaced until they reach a level where parasite growth and death are in equilibrium.

In the presence of a preference for reticulocytes, two competing parasite growth functions (occurring in erythrocytes and reticulocytes) contribute to a more complicated dynamic. 
Early in infection the proportion of reticulocytes is low, so the parasite and RBC curves are similar to those seen without a preference, and lead to an early peak of parasites in erythrocytes (green curve in Figure 3B). However, the depletion of RBCs during this time leads to increased production of the parasite's preferred target (reticulocytes). Thus, a second wave of parasite growth emerges, occurring predominantly in reticulocytes (red curve in Figure 3B). This wave of parasite invasion may lead to higher parasitemias than can be achieved by infection of older RBCs alone (compare Figure 3A and B).

\section{High Reticulocyte Production Increases Severity of Infection}

The above scenario describes how an increased preference for reticulocytes can drive higher parasitemias and lower RBC levels when combined with normal reticulocyte replacement rates. However, a similar scenario emerges when the reticulocyte replacement rate is varied in the presence of a fixed preference for reticulocyte invasion (Figure 4).

For low RRRs, the dynamics are primarily determined by the infection rate of the parasite in erythrocytes (since few reticulocytes are present at any given time) and we essentially see target cell limited growth of parasites in erythrocytes. Initially, the reproductive ratio of the parasite is greater than one and parasitemia increases. As target cells are depleted the reproductive ratio of the parasite drops to below one, and the parasite level begins to decrease again. We see a minimum in the number of uninfected RBCs followed by a slow recovery to an equilibrium level (Figure 4A).

At higher reticulocyte production rates, the presence of increased numbers of the preferred target later in infection can force the reproductive ratio of the parasite above one and lead to a second wave of reticulocyte infection, resulting in higher parasitemias and lower uninfected RBC levels (Figure 4B). Under certain circumstances this can lead to a biphasic appearance in the total parasite number (Figure 4C) similar to what is observed during experimental $P$. berghei infection (Figure 4D) $(25,32)$. Note that the time scales for the two plots are different since Figure $4 \mathrm{C}$ is a simulation of human infection, whilst Figure 4D depicts data from mice. A hump arising from parameters appropriate for the murine system and which occurs at a similar time to that in Figure 4D is shown in Supplementary Figure 9 of the SOM. This hump in parasite number has previously been attributed to the onset of acquired immunity (Singer, 1954b) or a shortage of reticulocytes (Janse and Waters, 2005), but here we show that it could simply be a result of reticulocyte replacement and preference.

We next investigate the combined impact of different levels of reticulocyte preference and reticulocyte production on the outcome of malaria infection. We focus on four key outcome measures: the lowest attained level of uninfected cells (minimum RBC, Figure 5 and Figure $6 \mathrm{~A}$ ), the long term uninfected RBC level (equilibrium RBC, Figure 6A), the maximum parasitemia (Figure 6B), and the long term (equilibrium) parasitemia (Figure 6B) during an infec- 
tion. The minimum uninfected RBC level is a marker of the degree of SMA generated by infection, and is a major cause of death during crisis. Maximum parasitemia can serve as a marker of hyper-parasitemia, again another cause of severe morbidity. The equilibrium levels of uninfected RBCs and of parasitemia are indicative of the long terms effects of infection, and can serve as markers for long term morbidity. In order to minimize the negative effects of disease, it would be advantageous to have both high minimum and equilibrium RBC levels, and low parasitemias.

\section{In the Presence of Reticulocyte Preference, Lower RRR Leads To Better Outcomes}

In the absence of a preference for reticulocytes $(p=1)$, increasing the RRR does not affect the equilibrium levels of uninfected RBCs (Supplementary Figure 2A). This is to be expected, as the equilibrium level of target cells is not affected by the production rate of targets (Nowak and Bangham, 1996). However, perhaps somewhat counter-intuitively, once the parasite has even a slight preference for reticulocytes, an increase in RRR always leads to lower equilibrium levels of uninfected red cells. This relationship can be seen in the dotted line in Figure 6A for a preference value of $p=5$. We see that as the RRR is increased, equilibrium RBC levels are decreased. We can also see this relationship clearly on the right hand side of Figure 7.

Raising the RRR causes a larger pool of reticulocytes to be created. Since the parasite has a preference for invading reticulocytes, this provides a large pool of the preferred target and hence boosts the number of parasitized cells. The result is that a large percentage of the newly created reticulocytes become parasitized and then rupture a short time later, without contributing to the overall RBC numbers. Furthermore, the increase in the number of parasitized cells results in more merozoites being produced which invade both mature RBCs and reticulocytes. Thus, not only are the newly created reticulocytes destroyed, but so are many older RBCs. The net result is that increasing the RRR in the presence of a preference of the parasite for reticulocytes leads to both an increase in equilibrium parasitemia and a decrease in equilibrium levels of uninfected RBCs (Figure 6). Therefore, in the presence of a reticulocyte preference it appears to be beneficial to have a RRR that is lower than normal.

\section{There Is a Threshold RRR That Minimizes RBC Loss In Acute Infection}

When there is no preference of the malaria parasite for reticulocytes $(p=1)$ then an elevated RRR allows cells to be replaced as they rupture, and so the minimum RBC level rises with increasing RRR (Figure 5). Once a preference of the parasite for reticulocytes $(p>1)$ is introduced then increasing the RRR no longer necessarily increases the minimum RBC levels (Figure 5). However, unlike the equilibrium RBC level, the minimal RBC level does not change monotonically with the RRR. In particular, there is an optimal RRR, which we call the threshold RRR at which the minimum RBC level reaches it highest value (Figure 5) and 
Figure 6A. This threshold divides the range of possible RRRs into two intervals, the first from the baseline RRR to the threshold, and the second from the threshold to the normal RRR. We observe qualitatively different types of behaviour in these:

Baseline to Threshold: The minimum RBC level increases slowly with increasing RRR (solid curve, left of Figure 6A). This region gives similar behaviour to that seen in the absence of a reticulocyte preference. It corresponds to the profile of infection depicted in Figure 4A and the dotted curve in Figure 7. Uninfected RBC levels decline to a minimum, which occurs close to the time that parasitemia reaches a peak. Since the RRR is still relatively low, there is only a small pool of target reticulocytes at this time point. Parasite levels are therefore controlled by a lack of availability of target cells. Gradual reticulocyte replacement then allows the RBC levels to increase from the minimum, while the parasite levels remain controlled. Increasing the RRR in this phase allows newly created reticulocytes to raise minimum RBC levels.

Threshold to Normal: An increase in RRR actually causes a decrease in the minimum RBC levels. The profile of an infection in this phase is depicted in Figure $4 \mathrm{~B}$ and the dashed curve in Figure 7. RBC levels decrease due to the initial rise in parasitemia. As the RBC levels decrease large numbers of reticulocytes are generated due to the elevated RRR. These reticulocytes provide new targets for the parasite to invade, and fuel the fire of infection from this point onwards. Almost all generated reticulocytes then become parasitized and thus cannot go on to mature into erythrocytes and cannot contribute to raising the RBC levels. This causes the RBC levels to decrease further (and the parasite levels to rise) to a lower minimum RBC level. There is no recovery of the RBC level and the minimum value now coincides with the equilibrium. In this range of RRR values therefore the patients reach an equilibrium with chronically high levels of reticulocyte production and parasitemia, and low levels of RBCs. Increasing the RRR during this phase only adds more target cells and further decreases the minimum RBC levels.

In order to maximize the minimum RBC level during the infection it is optimal to be at the threshold RRR on the boundary between these two intervals. The threshold RRR will vary with different reticulocyte preferences $p$. The minimum RBC level attained at this threshold RRR for each $p$ is shown by the black curve in Figure 5. As the reticulocyte preference $p$ increases the threshold RRR drops towards the baseline and the corresponding minimum RBC level also falls. Similar behaviour is seen for other infection rates $g$ (Supplementary Figure 1).

\section{Effect on Parasitemia Levels}

In the absence of a preference for reticulocytes $(p=1)$, an early rise in parasitemia is achieved due to the presence of a large number of erythrocytes in early infection (seen in the 
left of Figure 3A). An equilibrium RBC level is then reached where the density of RBCs allows the parasite numbers to be in equilibrium. In the presence of a preference for reticulocytes $(p>1)$, a similar early peak in parasitemia occurs, driven predominantly by the infection of erythrocytes, since reticulocyte numbers are low. This is seen as the initial peak in parasitized erythrocytes in Figure 3B and Figure 4A and B. Parasite numbers then decline as infected cells are destroyed.

If there are insufficient reticulocytes produced to push the basic reproductive rate of the parasite back above one, parasite numbers will continue to decrease, and the infection will be controlled (as is the case in Figure 4A). In this scenario the RRR does not have a significant effect on the peak parasitemia (flat section on left of solid curve in Figure 6B), which is far more sensitive to the reticulocyte preference and the natural rate of infection. However, in the presence of a high RRR, a burst of reticulocyte production occurs to replace the RBCs lost due to infection. If the RRR is elevated sufficiently so that a large pool of highly susceptible reticulocytes accumulates, then the reproductive ratio of the infection is pushed above 1 , and parasite numbers will begin to increase again, driven by newly produced reticulocytes. This can be seen in the second half of Figure 4B, which shows a second rise in parasite numbers, driven predominantly by parasitisation of reticulocytes. This is also seen on the left of the solid curve in Figure 6B where peak parasitemia starts to increase much more rapidly in response to rising RRR.

The critical RRR in terms of the peak parasitemia is the one which pushes the basic reproductive ratio of the parasite back above 1 after the initial peak in parasites is controlled. This critical RRR is indicated in Figure 6B for a reticulocyte preference $p=5$ and for a range of different preferences Suplementary Figure 2. This reveals that except for very low preferences, the critical RRR for parasitemia is higher than the threshold RRR for the minimum RBC. Hence, as long as the RRR is less than or equal to the threshold, unacceptably high parasitemias are not attained. This further reinforces the importance of having an RRR below the threshold.

Note also that the peak parasitemia obtained during an infection is highly sensitive to the parasite preference $p$ for reticulocytes. A high preference leads to a much higher peak parasitemia for a given RRR (Supplementary Figure 2). The peak parasitemia is also very sensitive to the rate of infection of the parasite in erythrocytes, with higher infection rates leading to higher peak parasitemias (data not shown).

\section{Estimating the Optimal Reticulocyte Replacement Rate}

The optimal RRR will be one which maximizes equilibrium red blood cell levels, minimizes equilibrium parasite numbers and does not force the patient to endure unacceptably low transient RBC levels or exceedingly high parasite levels. It will thus depend on what RBC level 
is classified as "unacceptably low", and how long a person can withstand lowered RBC levels during acute infection. If a patient is able to withstand a lower RBC level for a period of time then an RRR closer to baseline will be optimal as it will lead to increased equilibrium RBC levels, and lower equilibrium parasitemias. However, optimizing equilibrium RBC levels is not important if the patient is unable to survive the early transient period of severe anemia caused by a low RRR. It is also unclear that such an equilibrium state will ever be reached, since modifying factors such as immunity (which is not explicitly considered in the model) may help control long term infection. Thus, the optimal RRR lies somewhere between the threshold level (that produces the highest minimum RBC level) and baseline production (that produces highest equilibrium RBC level). An RRR just below threshold is often the best solution that minimizes the acute minimum of RBCs and also the time spent at this minimum, while still allowing a relatively high equilibrium level of RBCs. This is highlighted in Figure 7. Here the solid curve represents the course of infection with an RRR equal to the threshold value. The dotted curve, however, shows the course of infection using what is potentially the optimal RRR. We can see that RBC levels drop slightly below their threshold value for a short time, but the gain in terms of equilibrium RBC and parasitemia levels is substantial.

\section{DiSCUSSION}

The erythropoietic response to anemia during a malaria infection is not completely understood. It is often assumed to be suppressed below what would be expected in an uninfected individual, although the precise mechanism for this remains unclear. It has been shown that following clearance of the parasite, reticulocyte counts rise rapidly, sometimes by up to 10 fold, to combat the anemia (Abdalla et al., 1980). A priori, it would seem that erythropoietic suppression would hinder the recovery from anemia and would contribute to the pathogenesis of severe malarial anemia. However, in the presence of a preference of the parasite to infect reticulocytes, both the reticulocyte replacement rate and preferential invasion of reticulocytes strongly influence the outcome of an infection.

Studies involving iron have shown that incidence of malaria is lower among iron deficient children (Nyakeriga et al., 2004). We propose that a key reason for this is that the lower hemoglobin levels associated with iron deficiency can act as a protection against severe malaria. In the presence of a strong preference for reticulocytes newly created cells provide a target for infection and serve to boost parasite numbers. A strong reticulocyte response combined with a strong reticulocyte preference leads to catastrophic anemia and hyperparasitemia. Conversely, however, not producing enough new cells to replace cells dying due to parasitisation and rupture will lead to anemia in its own way by depleting the host of all target cells. 
In this paper, we have investigated the circumstances under which a suppressed reticulocyte response may in fact be advantageous to the host. We found that in the presence of a mild preference of the parasite for reticulocytes, such as that seen in $P$. falciparum, there is an intermediate RRR which balances the effects of target cell generation and host cell depletion to provide the most beneficial outcome to the patient. We call this intermediate rate the threshold RRR. The threshold RRR maximizes the minimum RBC level that the patient is forced to suffer and having an RRR above this threshold value confers no benefit to the patient. However lowering the RRR still further below the threshold value can help increase equilibrium RBC levels, and lower long term parasitemias. Thus the optimal reticulocyte replacement rate is one which is less than or equal to the threshold value.

We have not included an immune response of the host to the parasite in this analysis. It is still possible to gain valuable insights into infection dynamics while excluding an immune response, as has been shown in HIV (Phillips, 1996). However if we were to include an immune response, it is reasonable to assume that this would lower the overall growth rate of the parasite. The trends presented here would still be seen even if the rate of infection was lowered due to an immune response (Supplementary Figure 3). The only factors that change with a change in infection rate are the actual values of the threshold and optimal RRR. The only factors that change with a change in infection rate are the actual values of the threshold and optimal RRR. In addition, allowing for a delayed onset adaptive immune response that increases the clearance of infected cells also gives qualitatively similar results (results shown in supplementary materials).

The range of estimates of the reticulocyte preference, $p$, for $P$. vivax is 34-180. Under these conditions, we find that the threshold RRR is baseline production. However even for the range of preference estimates reported for P. falciparum (1.6-14 fold) the threshold RRR is close to baseline production levels. Increasing the RRR above this relatively low threshold value would actually lead to more severe anemia and hyperparasitemia. This is in agreement with the results of \{McQueen, $2004 \# 89$ \} which also showed that for parasite species that invade only reticulocytes, compensatory erythropoiesis can increase parasitemia. Thus the lowered reticulocyte replacement rates observed in many subjects (Abdalla et al., 1980; Abdalla, 1990) could actually be a host protective effect, with the host effectively seeking out the optimal reticulocyte replacement rate to minimize disease progression.

Erythropoietic suppression during malaria infection is often considered an inadvertent outcome of severe infection and inflammation, where inflammatory mediators produced in response to parasitemia suppress bone marrow function and worsen anemia (Abdalla, 1990; Clark and Chaudhri, 1988; Kwiatkowski et al., 1990). However, we suggest that under most circumstances the reduction in reticulocyte production observed in vivo paradoxically serves to maintain RBC levels by preventing the accumulation of large numbers of reticulocytes and 
an explosive second wave of infection in these cells (Figure 4). It is interesting to speculate whether prolonged exposure to infection and chronic anemia in endemic areas may serve to damp down the erythropoietic response to anemia, an effect that may ultimately contribute to non-specific resistance to infection.

A number of studies involving iron supplementation of children in developing countries have shown an increase in malaria related adverse effects and malaria risk (Sazawal et al., 2006; Smith et al., 1989), reviewed in (Iannotti et al., 2006; Oppenheimer, 2001). In addition, incidence of malaria is reportedly lower in mildly anemic (Michon et al., 2007) and iron deficient (Nyakeriga et al., 2004) children. Different reasons for the relationship between iron status and disease have been postulated: i) Lower iron levels limit parasite growth (Fritsch et al., 1987; Raventos-Suarez et al., 1982); ii) A defect in immunoglobulin production results from iron deficiency which protects against malaria (Nyakeriga et al., 2004); iii) Iron can block the synthesis of nitric oxide which in turn would contribute to pathogen death (Sazawal et al., 2006); iv) Iron supplementation causes increased levels of reticulocytes which are preferentially infected (Nyakeriga et al., 2004). The modelling results presented here lend support to this final hypothesis. Increased red blood cell production as a result of iron supplementation could lead to an increase in parasitemia and anemia. In contrast, the lower RBC production rates in iron deficient or mildly anemic children could result in an RRR closer to the optimal RRR presented in this paper, and so protect them from severe malarial episodes.

Additional support for the conclusion that a lower RRR can be protective against malarial anemia is found in a study showing that SMA is most prevalent in children at ages when the EPO response to anemia is highest (O'Donnell et al., 2007). A high EPO response to anemia should correlate with a high RRR. Therefore early in life children have a high RRR and this is when SMA is its most severe. As children mature their RRR decreases, and so does their susceptibility to SMA.

Further experimental work is required to study the erythropoietic response to anemia in infected and uninfected individuals in malaria endemic areas, and understand both reticulocyte production and its effects on parasitemia and disease outcome. Measurement of hemoglobin, reticulocyte and parasite levels during some of the iron supplementation studies could give the necessary data to validate the results obtained here through modelling.

\section{ACKNOWLEDGEMENTS}

We would like to thank Louis Schofield and Krystal Evans for providing the data on P. berghei infection used in Figure 4D. We would like to thank James Beeson for helpful comments on the manuscript. 
DC is funded by an Imperial College London Deputy Rector's Scholarship and JS is supported by the UK Biotechnology and Biotechnology and Biological Sciences Research Council (BBSRC) via the Centre for Integrative Systems Biology at Imperial College (CISBIC), BB/C519670/1. MPD is a Sylvia and Charles Viertel Senior Medical Research Fellow and this work was supported by a James S. McDonnell Foundation $21^{\text {st }}$ Century Research Award for Studying Complex Systems, and the NHMRC.

\section{AUTHORSHIP}

DC and MPD worked on the conception of the project, design of the model, and the writing of the manuscript. DC developed the mathematical model, provided mathematical and graphical analysis. JS provided input on the model development, oversaw the mathematical aspect of the project and revised and helped write the manuscript.

\section{REFERENCES}

Abdalla, S., Weatherall, D.J., Wickramasinghe, S.N., and Hughes, M., 1980. The anaemia of P. falciparum malaria. Br J Haematol 46, 171-83.

Abdalla, S.H., 1990. Hematopoiesis in human malaria. Blood Cells 16, 401-16; discussion 417-9.

Anderson, R.M., May, R.M., and Gupta, S., 1989. Non-linear phenomena in host-parasite interactions. Parasitology 99 Suppl, S59-79.

Belair, J., Mackey, M.C., and Mahaffy, J.M., 1995. Age-structured and two-delay models for erythropoiesis. Math Biosci 128, 317-46.

Chang, K.H., Tam, M., and Stevenson, M.M., 2004a. Modulation of the course and outcome of blood-stage malaria by erythropoietin-induced reticulocytosis. J Infect Dis 189, 735-43.

Chang, K.H., Tam, M., and Stevenson, M.M., 2004b. Inappropriately low reticulocytosis in severe malarial anemia correlates with suppression in the development of late erythroid precursors. Blood 103, 3727-35.

Clark, I.A., and Chaudhri, G., 1988. Tumour necrosis factor may contribute to the anaemia of malaria by causing dyserythropoiesis and erythrophagocytosis. Br J Haematol 70, 99-103.

Colijn, C., and Mackey, M.C., 2005. A mathematical model of hematopoiesis--I. Periodic chronic myelogenous leukemia. J Theor Biol 237, 117-32.

Cromer, D., Evans, K.J., Schofield, L., and Davenport, M.P., 2006. Preferential invasion of reticulocytes during late-stage Plasmodium berghei infection accounts for reduced circulating reticulocyte levels. Int J Parasitol 36, 1389-97. 
Dondorp, A.M., Angus, B.J., Chotivanich, K., Silamut, K., Ruangveerayuth, R., Hardeman, M.R., Kager, P.A., Vreeken, J., and White, N.J., 1999. Red blood cell deformability as a predictor of anemia in severe falciparum malaria. Am J Trop Med Hyg 60, 733-7.

Egan, A.F., Fabucci, M.E., Saul, A., Kaslow, D.C., and Miller, L.H., 2002. Aotus New World monkeys: model for studying malaria-induced anemia. Blood 99, 3863-6.

Ekvall, H., 2003. Malaria and anemia. Curr Opin Hematol 10, 108-14.

English, M., and Snow, R.W., 2006. Iron and folic acid supplementation and malaria risk. Lancet 367, 90-1.

Fritsch, G., Sawatzki, G., Treumer, J., Jung, A., and Spira, D.T., 1987. Plasmodium falciparum: inhibition in vitro with lactoferrin, desferriferrithiocin, and desferricrocin. Exp Parasitol $63,1-9$.

Garnham, P.C.C., 1966. Malaria Parasites and Other Haemosporidia. Blackwell Scientific Publications, Oxford.

Hardy, K., and Stark, J., 2002. Mathematical models of the balance between apoptosis and proliferation. Apoptosis 7, 373-81.

Hastings, A., and Palmer, M.A., 2003. Mathematics and biology. A bright future for biologists and mathematicians? Science 299, 2003-4.

Hegner, R., 1938. Relative frequency of ring-stage plasmodia in reticulocytes and mature erythrocytes in man and monkey. American Journal of Hygiene 27, 690-719.

Iannotti, L.L., Tielsch, J.M., Black, M.M., and Black, R.E., 2006. Iron supplementation in early childhood: health benefits and risks. Am J Clin Nutr 84, 1261-76.

Janse, C.J., and Waters, A.P., 2005. The Plasmodium berghei research model of malaria.

Kwiatkowski, D., Hill, A.V., Sambou, I., Twumasi, P., Castracane, J., Manogue, K.R., Cerami, A., Brewster, D.R., and Greenwood, B.M., 1990. TNF concentration in fatal cerebral, non-fatal cerebral, and uncomplicated Plasmodium falciparum malaria. Lancet 336, 1201-4.

Mackey, M.C., 1997a. Mathematical models of hematopoietic cell replication and control. Prentice Hall

Mackey, M.C., 1997b. Mathematical models of hematopoietic cell replication and control. Prentice Hall

Maggio-Price, L., Brookoff, D., and Weiss, L., 1985. Changes in hematopoietic stem cells in bone marrow of mice with Plasmodium berghei malaria. Blood 66, 1080-5. 
McQueen, P.G., and McKenzie, F.E., 2004. Age-structured red blood cell susceptibility and the dynamics of malaria infections. Proc Natl Acad Sci U S A 101, 9161-6.

Michon, P., Cole-Tobian, J.L., Dabod, E., Schoepflin, S., Igu, J., Susapu, M., Tarongka, N., Zimmerman, P.A., Reeder, J.C., Beeson, J.G., Schofield, L., King, C.L., and Mueller, I., 2007. The risk of malarial infections and disease in Papua New Guinean children. Am J Trop Med Hyg 76, 997-1008.

Mons, B., 1990. Preferential invasion of malarial merozoites into young red blood cells. Blood Cells 16, 299-312.

Mons, B., Croon, J.J., van der Star, W., and van der Kaay, H.J., 1988a. Erythrocytic schizogony and invasion of Plasmodium vivax in vitro. Int J Parasitol 18, 307-11.

Mons, B., Collins, W.E., Skinner, J.C., van der Star, W., Croon, J.J., and van der Kaay, H.J., 1988b. Plasmodium vivax: in vitro growth and reinvasion in red blood cells of Aotus nancymai. Exp Parasitol 66, 183-8.

Nowak, M.A., and Bangham, C.R., 1996. Population dynamics of immune responses to persistent viruses. Science 272, 74-9.

Nyakeriga, A.M., Troye-Blomberg, M., Dorfman, J.R., Alexander, N.D., Back, R., Kortok, M., Chemtai, A.K., Marsh, K., and Williams, T.N., 2004. Iron deficiency and malaria among children living on the coast of Kenya. J Infect Dis 190, 439-47.

O'Donnell, A., Premawardhena, A., Arambepola, M., Allen, S.J., Peto, T.E., Fisher, C.A., Rees, D.C., Olivieri, N.F., and Weatherall, D.J., 2007. Age-related changes in adaptation to severe anemia in childhood in developing countries. Proc Natl Acad Sci U S A 104, 9440-4.

Oppenheimer, S.J., 2001. Iron and its relation to immunity and infectious disease. J Nutr 131, 616S-633S; discussion 633S-635S.

Pasvol, G., Weatherall, D.J., and Wilson, R.J., 1980. The increased susceptibility of young red cells to invasion by the malarial parasite Plasmodium falciparum. Br J Haematol 45, 28595.

Phillips, A.N., 1996. Reduction of HIV concentration during acute infection: independence from a specific immune response. Science 271, 497-9.

Raventos-Suarez, C., Pollack, S., and Nagel, R.L., 1982. Plasmodium falciparum: inhibition of in vitro growth by desferrioxamine. Am J Trop Med Hyg 31, 919-22.

Sazawal, S., Black, R.E., Ramsan, M., Chwaya, H.M., Stoltzfus, R.J., Dutta, A., Dhingra, U., Kabole, I., Deb, S., Othman, M.K., and Kabole, F.M., 2006. Effects of routine prophylactic 
supplementation with iron and folic acid on admission to hospital and mortality in preschool children in a high malaria transmission setting: community-based, randomised, placebocontrolled trial. Lancet 367, 133-43.

Singer, I., 1954a. The effect of splenectomy or phenylhydrazine on infections with Plasmodium berghei in the white mouse. J Infect Dis 94, 159-63.

Singer, I., 1954b. The course of infection with Plasmodium berghei in inbred CF 1 mice. J Infect Dis 94, 237-40.

Smith, A.W., Hendrickse, R.G., Harrison, C., Hayes, R.J., and Greenwood, B.M., 1989. The effects on malaria of treatment of iron-deficiency anaemia with oral iron in Gambian children. Ann Trop Paediatr 9, 17-23.

Stark, J., and Hardy, K., 2003. Mathematics. Chaos: useful at last? Science 301, 1192-3.

Wilson, R.J., Pasvol, G., and Weatherall, D.J., 1977. Invasion and growth of Plasmodium falciparum in different types of human erythrocyte. Bull World Health Organ 55, 179-86.

Zuckerman, A., 1957. Blood loss and replacement in plasmodial infections. I. Plasmodium berghei in untreated rats of varying age and in adult rats with erythropoietic mechanisms manipulated before inoculation. J Infect Dis 100, 172-206. 


\section{LEGEND TO FIGURES}

\section{Figure 1 Model of malaria infection kinetics.}

Processes included in the model are red blood cell production, parasitisation and destruction as well as merozoite creation, invasion and death. Reticulocytes $\left(\mathrm{R}_{\mathrm{U}}\right)$ enter the system from the top left in an uninfected state. These cells can either be cleared due to natural processes, become parasitized $\left(\mathrm{R}_{\mathrm{p}}\right)$ through contact with a merozoite $(\mathrm{m})$, or mature into an erythrocyte $\left(\mathrm{E}_{\mathrm{U}}\right)$. Erythrocytes can also become parasitized $\left(\mathrm{E}_{\mathrm{p}}\right)$ through contact with a merozoite. Parasitized cells rupture, producing merozoites, which then either decay, or go on to parasitize more cells.

\section{Figure 2 Examples of intermediate RRR curves.}

Curves show reticulocyte replacement rate curves for various intermediate values between baseline and normal production. Curves show number of reticulocyte produced per $\mathrm{ml}$ of blood volume in a single day, given different levels of anemia. Baseline production is a flat line. Normal RRR is given by the highest curve (maximum of 10 times baseline). Reduced RRR show intermediate maximum production rates at low RBC numbers.

Figure 3 Graphs showing the course of infection for different reticulocyte preferences in the presence of normal reticulocyte replacement.

Pink curves are plotted on left hand axes and show RBC counts as a percentage of normal RBC. Parasite curves are plotted on right hand axes and give number of parasitized cells $/ \mathrm{ml}$. Total parasite numbers (blue) are a sum of parasitized reticulocytes (red) and parasitized erythrocytes (green).

A) Normal reticulocyte production and equal invasion of erythrocytes and reticulocytes by the parasite;

B) Normal reticulocyte production and a 15 fold preference of the parasite for invading reticulocytes. Initial parasite growth is in erythrocytes, leading to a peak in erythrocyte parasitemia. This is followed by wave of parasites in reticulocytes once sufficient numbers of them have been produced.

Figure 4 Graphs showing effect of altering RRR in the presence of a preference for reticulocytes.

Legend is as for Figure 3, except in Figures C and D, parasite curves are plotted on the left hand axis.

A) Baseline reticulocyte production and a 15 fold preference of the parasite for invading reticulocytes. Since there are never a large number of reticulocyte present for the parasite to 
invade, the preference of the parasite for reticulocytes does not cause a significant rise in parasitemia;

B) Elevated reticulocyte production ( 6 fold increase from baseline) and a preference value of $\mathrm{p}=15$ of the parasite for invading reticulocytes. This scenario is similar to the one shown in $\mathrm{B}$, but we can see a hump in parasitemia which arises as we switch from seeing more parasites in erythrocytes to seeing more parasites in reticulocytes;

C) Enlargement of the section in Figure 4B centred at the first hump in parasitized RBCs;

D) Data for P. berghei infected mice adapted from (Cromer et al., 2006).

Figure 5 Effect of RRR and reticulocyte preference on minimum levels of unparasitized cells.

For a fixed reticulocyte preference initially the minimum RBC level attained increases with increasing RRR. It reaches a maximum at the threshold RRR, marked with a black dotted line and thereafter further increases in RRR lead to a rapid drop in the minimum unparasitized RBC level. The blue starred line indicates a reticulocyte preference of 5 and corresponds to the plot in Figure 6A.

Figure 6 Effect of altering RRR on four key features.

Dotted lines are equilibrium cell levels, solid lines are minimum level of unparasitized cells (A) and maximum level of parasitized cells (B).

A) Minimum and equilibrium unparasitized RBC levels for a reticulocyte preference of 5 and varying RRRs. The minimum RBC curve corresponds to the starred blue line marked in Figure 5. Also indicated is the threshold RRR and the corresponding minimum RBC level. B) Maximum and equilibrium parasitemias for a reticulocyte preference of 5. Also indicated is the critical RRR for maximum parasitemia.

Figure 7 Unparasitized RBCs as a percentage of the original RBC count for three reticulocyte replacement rates and a reticulocyte preference of 5. 


\section{TABLES}

\begin{tabular}{|l||l||}
\hline Symbol & Description (at time t) \\
\hline \hline$H(t)$ & Total Red Blood Cells $(H$ for Hemoglobin) \\
\hline$R_{u}(t)$ & Unparasitized reticulocytes \\
\hline$R_{u, \text { para }}(t)$ & Unparasitized reticulocytes becoming parasitized \\
\hline$R_{u, \text { mat }}(t)$ & Unparasitized reticulocytes maturing into erythrocytes \\
\hline$R_{u, \text { decay }}(t)$ & Unparasitized reticulocytes decaying \\
\hline$R_{p}(t)$ & Parasitized reticulocytes \\
\hline$R_{p, \text { mat }}(t)$ & Parasitized reticulocytes maturing into parasitized erythrocytes \\
\hline$R_{p, \text { rupt }}(t)$ & Parasitized reticulocytes rupturing \\
\hline$R_{p, \text { decay }}(t)$ & Parasitized reticulocytes decaying \\
\hline$E_{u}(t)$ & Unparasitized erythrocytes \\
\hline$E_{u, \text { para }}(t)$ & Unparasitized erythrocytes becoming parasitized \\
\hline$E_{u, \text { decay }}(t)$ & Unparasitized erythrocytes decaying \\
\hline$E_{p}(t)$ & Parasitized erythrocytes \\
\hline$E_{p, \text { rupt }}(t)$ & Parasitized erythrocytes rupturing \\
\hline$E_{p, \text { decay }}(t)$ & Parasitized erythrocytes decaying \\
\hline$M(t)$ & Merozoites \\
\hline$M_{\text {created }}(t)$ & Merozoites created \\
\hline$M_{\text {used }}(t)$ & Merozoites used up \\
\hline$M_{\text {decay }}(t)$ & Merozoites decaying \\
\hline$s\left(t_{b}, t\right)$ & Unparasitized cells born at time $t_{b}$ \\
\hline$c\left(t_{b}, t_{p}, t\right)$ & Parasitized cells born at time $t_{b}$ and parasitized at time $t_{p}$ \\
\hline \hline
\end{tabular}

Table 1 Variables used in the model 


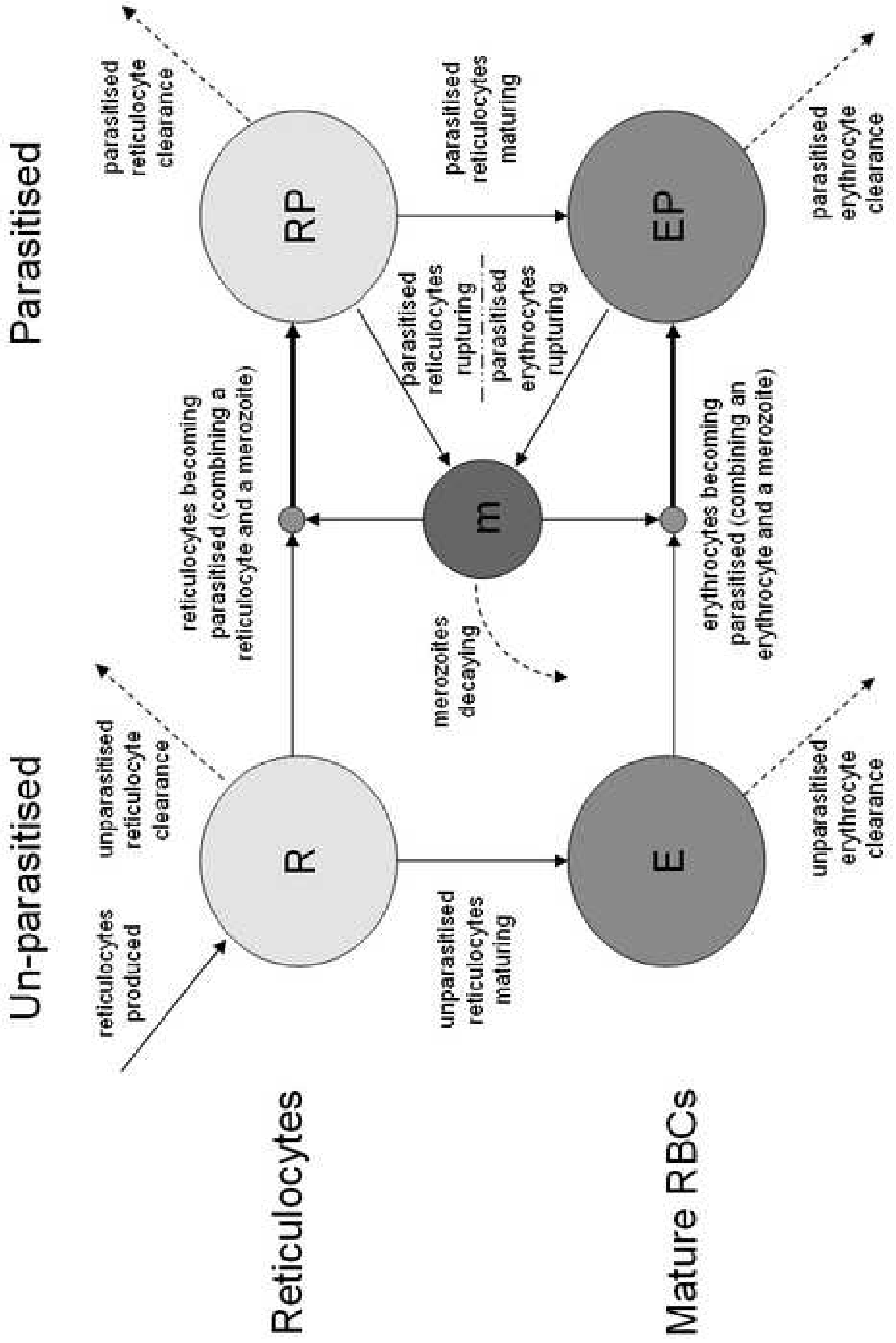



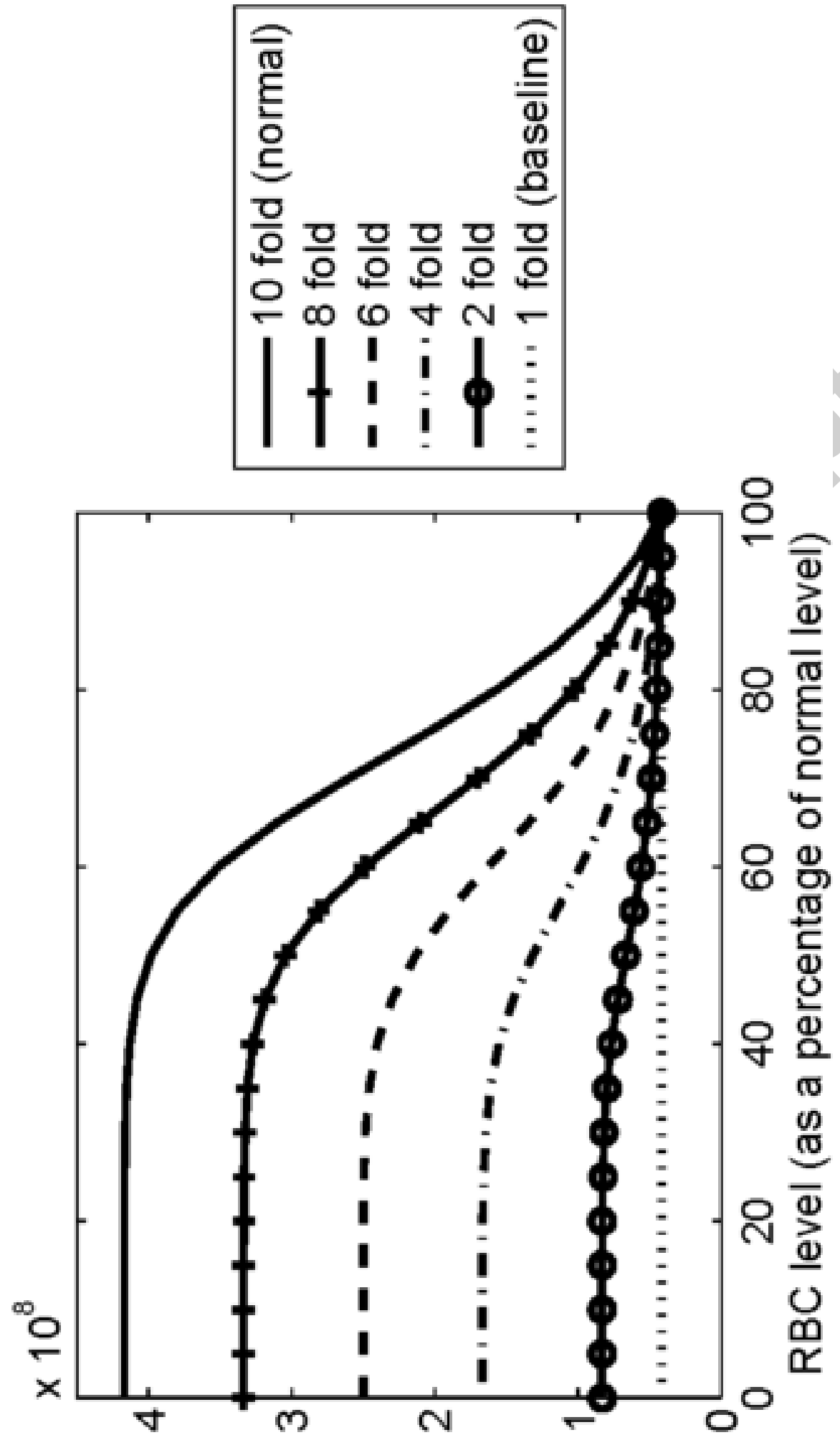

i_ Кep 


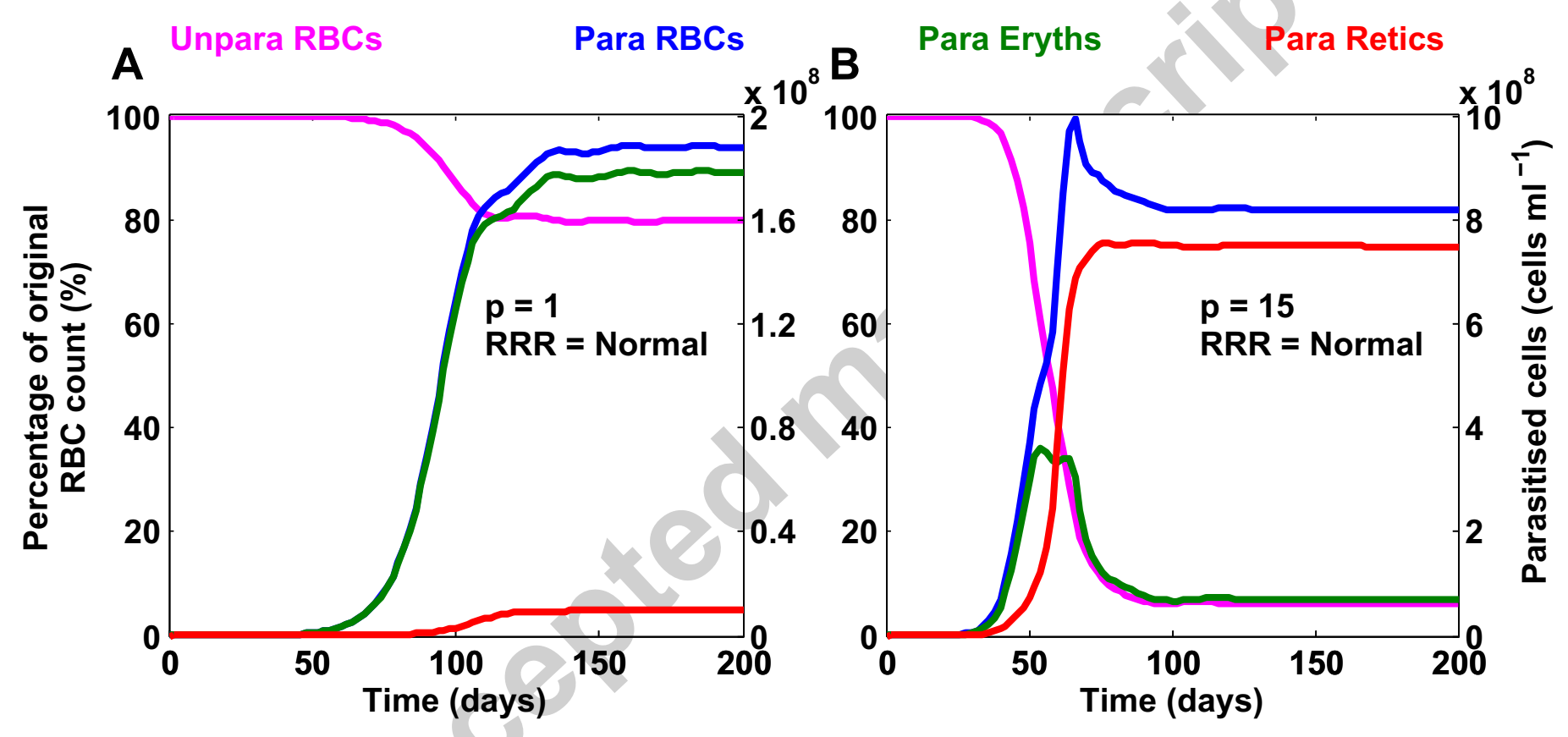



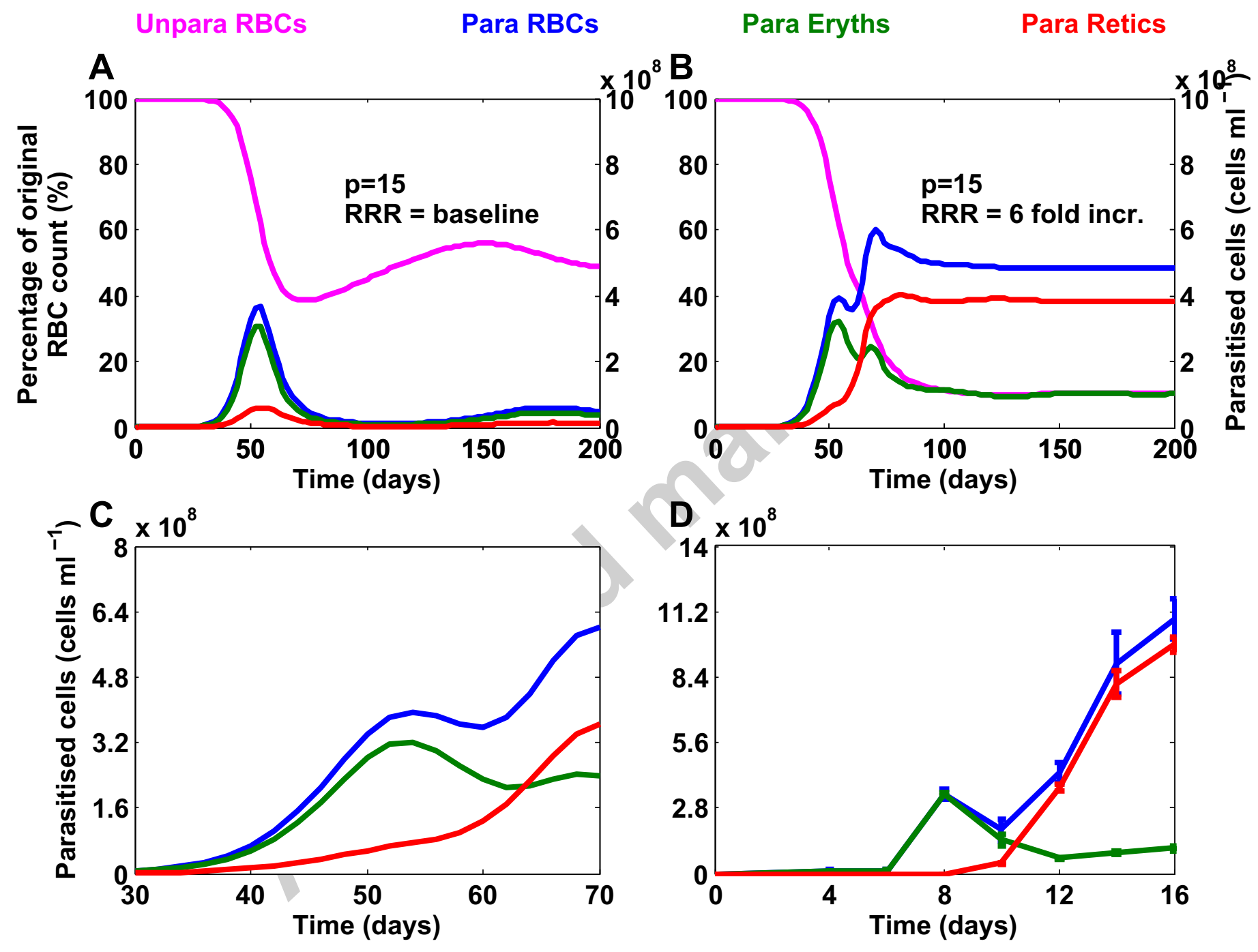


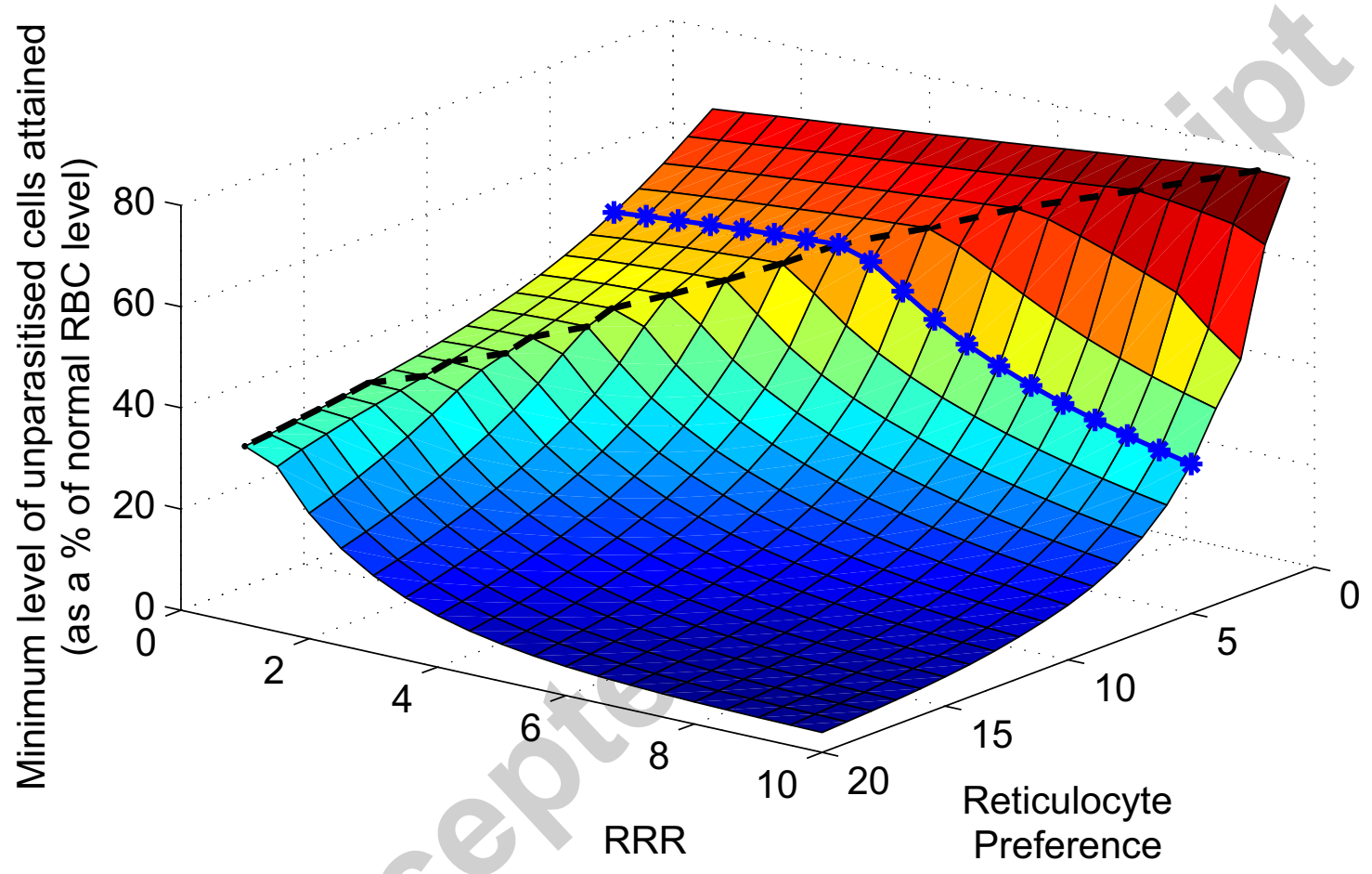


Figure 6 Effect of altering RRR on four key features
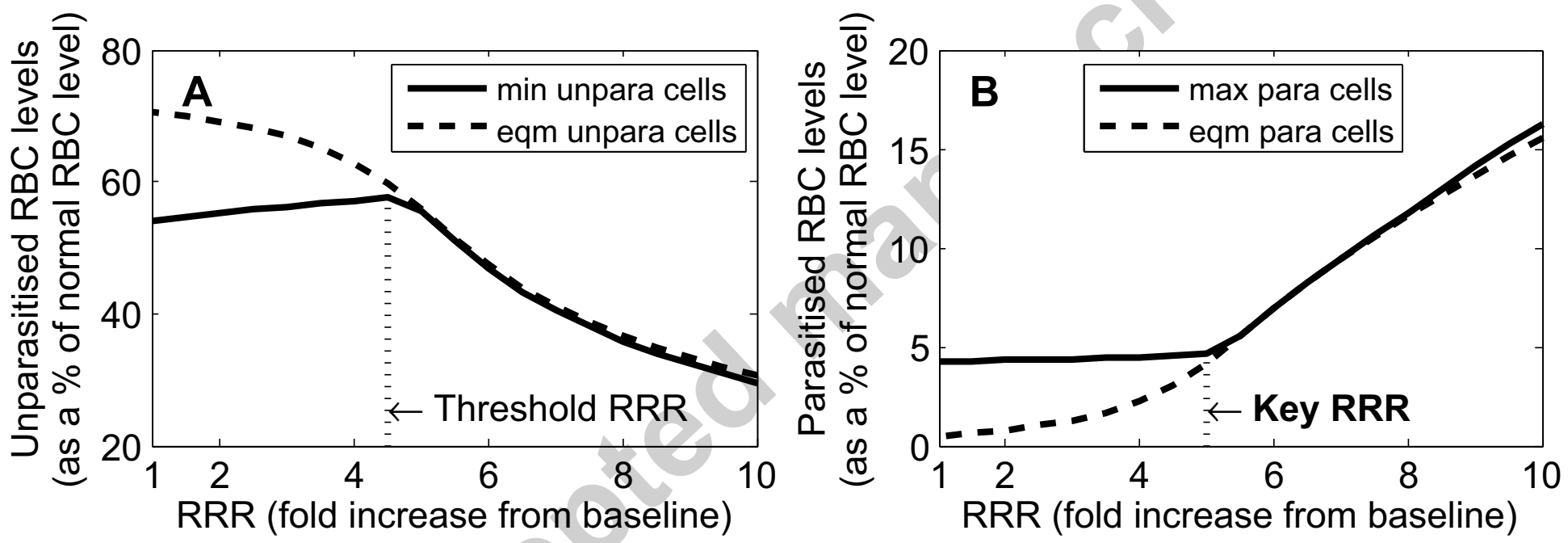
Figure 7 Unparasitized RBCs for three RRRs and a preference of 5

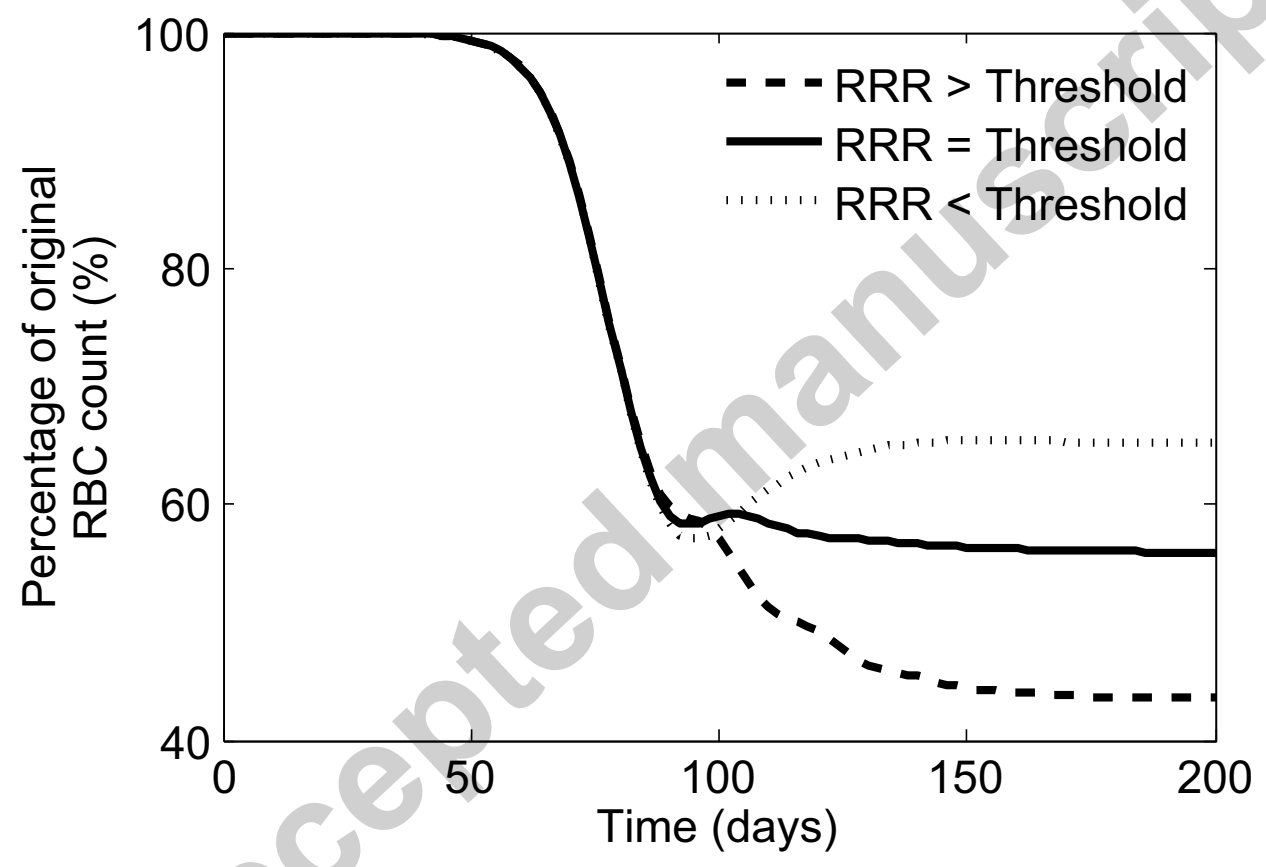

